\title{
An attempt to define perceptive and sensitive mapping through lived space experiments
}

\author{
Catherine Dominguès ${ }^{\mathrm{a},} *$, Laurence Jolivet ${ }^{\mathrm{a}}$, Éric Mermet $^{\mathrm{b}}$, Sevil Seten $^{\mathrm{c}}$ \\ ${ }^{a}$ LASTIG, Univ Gustave Eiffel, ENSG, IGN, F-94160 Saint-Mandé, France, laurence.jolivet@ign.fr; catherine.domingues@ign.fr \\ ${ }^{b}$ Centre d'analyse et de mathématique sociales (CAMS), École des hautes études en sciences sociale, eric.mermet@ehess.fr \\ ${ }^{c}$ Laboratoire Ambiances Architectures Urbanités, École doctorale STT de Nantes, sevilseten@gmail.com \\ * Corresponding author
}

Keywords: Perceptive and Sensitive Map, Sensitive Map, Mapping Experiment, Mapping Need

Maps are often used in the context of human and social sciences, including as a tool. For example, maps as graphic tools enable to locate survey fields and data. Especially, the synoptic property of maps makes it possible to investigate the spatial dimension of a phenomenon, the distribution of data, its changes over time, etc.

In teaching activities and in support tasks for research at the EHESS ${ }^{1}$ in Paris, difficulties have arisen in showing research data and results in a manner which would be fruitful and acceptable to the students and researchers.

The need for an adapted mapping has emerged, including the map-making process and the achieved map. Adapted mapping has been named by the phrase perceptive and sensitive mapping, in contrast with conventional mapping based on geographical databases, GIS tools and the theory of graphic semiology as taught by Jacques Bertin (Bertin, 1983).

In response to this need, a training methodological seminar has been set up since 2016 in EHESS. It aims at providing an (organizational and material) framework for students in which they can experiment various protocols and be confronted with different data specifications. The procedures are designed in order to accentuate specific aspects that are not supposed to be fulfilled by conventional mapping. An analysis has been performed targeting the students' achievements and how they have been achieved. The analysis makes it possible to characterize the maps drawn in this context; to compare the students' difficulties and comments with the needs they initially expressed; to highlight in which cases conventional cartography may be inadequate for laying out some data. The result analysis enabled considering three questions: how may conventional mapping and perceptive and sensitive mapping be compared? How is perceptive and sensitive mapping a relevant tool? And thanks to the answers of the previous questions: What would be a definition of perceptive and sensitive mapping?

To this end, the paper firstly details how the needs for maps were expressed and how the seminar tried to answer them by defining experiments. In the second section, the achievements are analyzed based on two items: the (displayed) graphical and cartographic features, and the protocols which enabled to make them. Lastly, the analysis enables to offer a definition of perceptive and sensitive mapping by means of a comparison with conventional mapping.

\section{Needs for maps}

A map is a technical object, created from its designer's intention and delivering a message to its readers, meeting more or less the designer's intention. Conventional cartography uses graphic semiology; it focuses on the efficiency of the cartographic message (conveyed by the symbols) which will be read and must be adequately understood and remembered. As a counterpart to the definition of symbols verifying visual variables and to the observance of rules, it "guarantees" the efficiency of the map which is supposed to deliver an unambiguous message. Some data do not fit well with the conventional mapping rules, for example: perceptive data from the five senses which are not quantifiable, short-lived or not repeatable; sensitive data (as emotions or opinions) linked to places; non geographical data such as certain explanatory variables of a phenomenon which must be explained. The concepts and rules on which conventional mapping is based are proved to be inadequate, for example: in (Olmedo, 2016) concerning life stories; in (Mekdjian, 2016), (Mekdjian \& Olmedo, 2016) migration narratives; in (Bacon et al., 2016) migratory movements; in (Rosemberg \& Troin, 2017) mapping literature. Adaptations are necessary to be able to represent these data. They can be spontaneous or use prior knowledge of cartography and graphic semiology. They are guided by the uses of the mapmakers' previously produced artifacts: notebook, collective mapping cartography, and by different focuses: interest of spatial representation as field notebooks, backing for dialogue with field practitioners, etc.

\section{Analyses of perceptive and sensitive maps}

The maps created by the seminar participants (an example is detailed in Figure 1) were analyzed with reference to the steps and rules of a simplified conventional map-making process, which is taught in particular in mapping courses. The process is developed over six steps:

\footnotetext{
${ }^{1}$ The EHESS is the École des Hautes Études en Sciences Sociales, the School of Advanced Studies in the Social Sciences. It trains students up to $\mathrm{PhD}$ level in all disciplines of the social and human sciences (history, sociology, geography, linguistics, etc.).
} 
1/ Analysis of the map goals: the message of the map is not based on a specific point of view which would be prioritized in the totality of the map. Rather, a profusion of points of view are visible depending on the various represented zones. Hence, the information display does not contribute to their ranking;

2/ Definition of the map border: previous thinking about size and scale is not a prerequisite for mapping. Consequently, the scale is not homogeneous in all the directions; drawing is done simultaneously to the identification and selection of data; the overall topography of the mapped area does not match the physical reality;

3/ Selection of located items: the selection of the data to be mapped is not homogeneous in the whole map, since the elements considered as salient and therefore represented are selected at the time of the experiment. These data reflect the experienced space in a spontaneous way;

4/ Definition of symbols: displaying of items which are difficult to locate or non-visual, such as the sound of a plane, leads to define symbols that are not always explicit. Also, the level of detail is not homogeneous: some items may be very detailed and others not; items may be displayed in some parts of the map, and others not even if they belong to the same themes; some of them are represented partially, only by their intersection with some points of interest. In general, the maps show a lot of details, these salient elements testify of the interpretation by the map-maker of the visited area according to its personal patrimonial, social or landscape interests (Rosemberg \& Troin, 2017);

5/ Map drawing: data drawing is concomitant to their selection. The selection and the display of the data present in the map are done simultaneously. The map is the material for both warehousing and displaying the perceptive and sensitive data, unlike in the conventional mapping process where data are stored in databases and then selected according to the purpose of the map for display;

6/ The displaying adaptations which are observed on the examples of perceptive maps produced by the students (liberty with the metric, adaptive and non-constant scale, non-homogeneous selection of data) are to be related to the difficulties encountered in the map evaluation. The evaluation of their effectiveness needs to be carried out according to the context of their use and, in particular, to the objective of conveying emotions, opinions, perceptions, feelings, attached to places.

a)

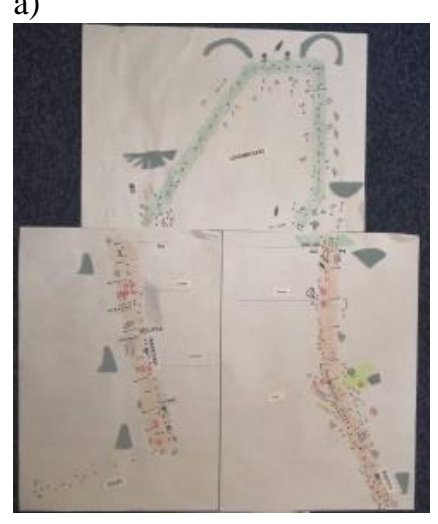

b)

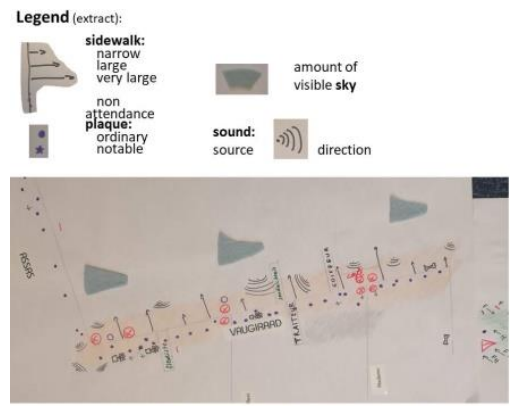

c)

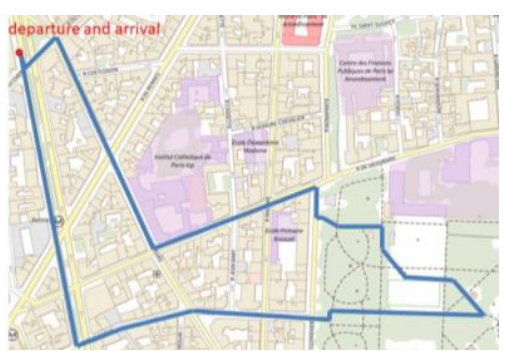

Figure 1. The figure illustrates an urban walk around the EHESS building in Paris (France). Each participant chose a filter (sidewalks, sounds, plaques, etc.) and experienced the space through this filter. In a) is the collective map which gathers all the filters; in b) are excerpts from the map and the legend; the map in c) shows the effective itinerary (https://www.geoportail.gouv.fr/)

\section{An attempt to define perceptive and sensitive mapping}

Perceptive and sensitive mapping claims to represent an experience of the territory, and because reality is complex and inaccessible, it accepts a partial and contextualized interpretation of the territory. Mapping space as lived space is first of all linked to its conditions of implementation: in this approach, the person who experiments is also the one who represents; so the approach is intended to be spontaneous and intuitive and is not preceded by an attempt to distance from the object or phenomenon to be perceived and represented. The purpose is to display a perceptive experience of space that relies on the five senses and a sensitive experience that bears witness to emotion, feeling or opinion. Sensitive experience is based on present experience but can also be influenced by prior knowledge, connotations and memory, for example regarding the living space display during lockdown (Jolivet et al., 2021). In addition, the artistic dimension is not absent from this mapping practice.

These presuppositions induced by the experiments lead to take into account a) perceptive or emotional data that are not relevant to precisely locate and delimit because of their very nature (for example, the intermittent presence of car horns involved in the predictability of a sound and therefore in the sound pleasantness of a place); and b) data which are not homogeneous or whose granularity is variable because they depend on sensations and perceptions which are necessarily incomplete and partial. This means that different points of view can coexist on the same map. The reading of the map can thus become ambiguous, but this ambiguity is accepted instead of being masked by the map's status as a scientific object. 


\section{References}

Bacon, L., Clochard, O., Honoré, T., Lambert, N., Mekdjian, S. and Rekacewicz, P. (2016). Cartographier les mouvements migratoires. Revue européenne des migrations internationales, vol. 31, n³, p. 185-214.

Bertin, J., Berg, W.J. and Wainer, H. (1983). Semiology of graphics: diagrams, networks, maps. University of Wisconsin Press Madison.

Jolivet, L., Dominguès, C., Mermet. É. and Seten, S. (2021). Living space as used and perceived during lock-down in a sensitive map approach. $30^{\text {th }}$ International Cartographic Conference ICC'2021, December 14-18, Florence, Italy (accepted).

Mekdjian, S. (2016). Les récits migratoires sont-ils encore possibles dans le domaine des Refugee Studies ? Analyse critique et expérimentation de cartographies créatives. In : ACME: An International E-Journal for Critical Geographies 15.1.

Mekdjian, S. and Olmedo, É. (2016). Médier les récits de vie. Expérimentations de cartographies narratives et sensibles. In: M@ppemonde 118. URL: https://halshs.archives-ouvertes.fr/halshs-01242536.

Olmedo, É. (2016). Pour une cartographie affective des récits des femmes de Sidi Youssef Ben Ali (Marrakech, Maroc). In: Cartographier les récits. Sous la dir. de Mauricette Fournier. CERAMAC. Clermont-Ferrand : Presses universitaires Blaise Pascal, p. 179-196.

Rosemberg, M. and Troin, F. (2017). Cartographie du Marseille d'un héros de roman policier Total Khéops de J.-C. Izzo. In: M@ppemonde 121. URL: http://mappemonde.mgm.fr/121_as2/. 